\title{
Macroscopic Thin Film Deposition Model for the Two-Reactive-Gas Sputtering Process
}

\author{
András KELEMEN ${ }^{1}$, Domokos BIRÓ ${ }^{2}$, Albert-Zsombor FEKETE ${ }^{1}$, \\ László JAKAB-FARKAS ${ }^{1}$, Róbert Rossi MADARÁSZ ${ }^{1}$ \\ ${ }^{1}$ Department of Electrical Engineering, Faculty of Technical and Human Sciences, \\ Sapientia Hungarian University of Transylvania, Tg. Mureş, \\ e-mail:kandras@ms.sapientia.ro \\ ${ }^{2}$ Department of Mechanical Engineering, Faculty of Technical and Human Sciences, \\ Sapientia Hungarian University of Transylvania, Tg. Mureş, \\ e-mail: dbiro@ms.sapientia.ro
}

Manuscript received February 15, 2017; revised March 15, 2017.

\begin{abstract}
The presence of a second reactive gas in the magnetron sputtering chamber makes the process much more complicated, and the process control much more difficult than in the case of a single reactive gas. Macroscopic models have been developed in order to explain the complex phenomena and to provide support for the process control. These models are able to explain the nonlinearities of the process and the strong coupling between the control channels.

This paper introduces a model created with the intention to of gaining a good grasp of the process, especially regarding the conditions necessary to obtain the required stoichiometry of the film deposited on the substrate. For this purpose, we modelled the formation of the desired ternary compound both directly from the available particle fluxes and from intermediary compounds. The surface of the substrate is divided into eight dynamically variable regions, covered by different compounds, each exposed to the streams of five types of particles.

We present the analytical model and provide simulation results in order to demonstrate its capability toof describeing the nonlinear phenomena, which that characterisze the two-gas sputtering process.
\end{abstract}

Keywords: DC magnetron sputtering, thin film deposition, reactive sputtering, macroscopic modelling.

\section{Introduction}

DC magnetron sputtering and thin film deposition have been studied for decades and several attempts have been made for to modellling different aspects of these processes. 
There exist several works dedicated to the study of the reactive DC magnetron sputtering process, which basically consists of sputtering particles from the surface of a metallic target, in the presence of reactive gases. The sputtering effect is produced by Ar+ ions accelerated by a DC electric field.

A successful approach for to macroscopic modeling was provided by Berg et al. [1], [2], which is based on the equilibrium equations of the reactive gas and metal particles on the target and substrate surfaces. This model has been successful in explaining the strong nonlinearity and hysteresis effects observed in the process, and is simple enough to be used for process control.

Since then, a number of extensions of this idea have been formulated, to handle the cases wheren compound targets or more reactive gases are being used ([2], [11], [12], [13], [14]). Dynamic versions of these models have been formulated for control purposes ([7], [8], [9], [10], [15]).

In the presence of two reactive gases, the simplified modelling approach generally used is to consider, instead of the complex crystallization phenomena, only binary compounds $M G_{1}$ and $M G_{2}$ (ex. $M G_{1} \equiv T i O_{2}, M G_{2} \equiv T i N$ ), the participating average metal and gas quantities reflecting the stoichiometry of the real process.

This paper attempts to provide a more detailed macroscopic model of the thin film growth process by highlighting its intermediary phases. Thus, we assumed that the surface of the substrate consisted of areas covered by metal atoms $(M)$, areas covered by reactive gas atoms $\left(G_{1}\right.$ and $\left.G_{2}\right)$, areas covered by binary metal-gas compounds $\left(M G_{1}\right.$ and $\left.M G_{2}\right)$ and by ternary $M G_{1} G_{2}$ compounds (Fig. 1). The coverage fractions represented by these areas are changing vary due to the incident particle fluxes and due to the bonds formed between the particles adsorbed to the surface of the substrate.

To some extent, the process is can be thought of as if different coloureds of paints were applied on to a surface, using atomizers.

Regarding the phenomena on the surface of the target, it is assumed that the Ar+ ion flux can sputter both metal atoms (ex. Ti) and molecules formed from this metal and the atoms of the reactive gases (ex. N, O). We used a single layer approach, i.e. removingal of an oxide or nitride molecule results in to leaves behind a metallic surface.

The target fractions covered by different compounds are denoted by $\theta_{t i}$, i.e.

$\theta_{t M}$ - the metallic fraction of the target surface;

$\theta_{t M G 1}, \theta_{t M G 2}$ - the fractions of the target surface ,poisoned” by $M G_{1}$ and $M G_{2}$ binary compounds

Formation of ternary compounds was not taken into account on the surface of the target. 
In the case of the substrate, the fractions covered by different compounds were denoted by $\theta_{s i}$, where $i \in\left\{M, G_{1 a}, G_{2 a}, M G_{1 a}, M G_{2 a}, M G_{1 p}, M G_{2 p}, M G_{1} G_{2}\right\}$, i.e.

$\theta_{S M}$ - the metallic fraction of the substrate surface;

$\theta_{S M G 1 G 2}$ - the fraction covered by the ternary compound $M G_{1} G_{2}$;

$\theta_{S M G 1 a}, \theta_{S M G 2 a}$ - the fractions covered by the ,active" binary compounds $M G_{1}$ and $M G_{2}$ (formed on the surface of the substrate from incident atomic components, ready available to react with $G_{2}$ or $G_{1}$ respectively, considered as intermediateas intermediate phases of the $M G_{1} G_{2}$ crystal growth);

$\theta_{S M G 1 p}, \theta_{S M G 2 p}$ - the fractions covered by the ,passive" binary compounds $M G_{1}$ and $M G_{2}$ (resulted fromby sputtering of the same compounds from the surface of the target, expected to segregate on the grain boundary);

$\theta_{s G 1 a}, \theta_{s G 2 a}$ - the fractions covered by the ,active" gas atoms $G_{1}$ and $G_{2}$

(formed from incident gas atoms, ready to participate in the crystal growth, forming directly $M G_{1} G_{2}$, or ,active" $M G_{1}$ and $M G_{2}$ compounds).

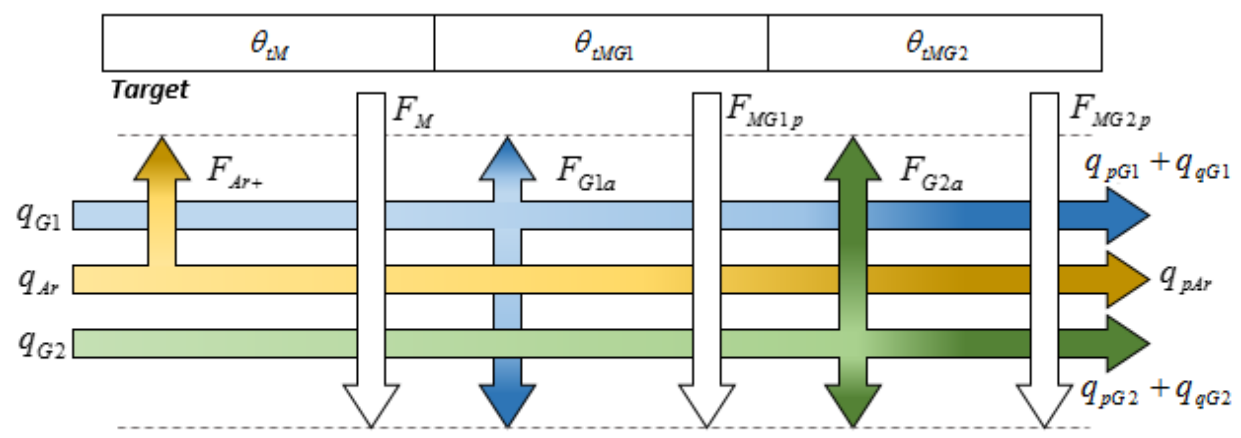

Substrate

\begin{tabular}{|c|c|c|c|c|c|c|c|}
\hline$\theta_{\text {SMGG }}$ & $\theta_{\text {SMGIa }}$ & $\theta_{\text {SMG2 }}$ & $\theta_{\text {SGIa }}$ & $\theta_{\text {SG2 }}$ & $\theta_{\text {SMG1 } p}$ & $\theta_{\text {SMG2 }}$ & $\theta_{\text {SM }}$ \\
\hline
\end{tabular}

Figure 1: Particle fluxes in the sputtering chamber and coverage fractions on the target and substrate surfaces

The deposition of metal atoms on the metallic fraction does not influence the $\theta_{s M}$ fraction. In the same manner, deposition of $G_{i}$ on $\theta_{s G i a}$ does not change $\theta_{s G i a}$, deposition of $M G_{i}$ on $\theta_{s M G i p}$ does not change $\theta_{s M G i p}$. A reaction, which yields $M G_{i}$ on the $\theta_{s M G i a}$ fraction (using $M$ and $G_{i}$ deposited on the same fraction), does not modify $\theta_{s M G i a}$, and a reaction which yields $M G_{1} G_{2}$ on the 
$\theta_{S M G 1 G 2}$ fraction (using $M, G_{1}$ and $G_{2}$ deposited on $\theta_{S M G 1 G 2}$ ), does not modify $\theta_{S M G 1 G 2}$.

In this paper we assumed that different compounds are formed only on the surface of the substrate, and the formation of compounds in the plasma was neglecteddisregarded. We considered that the reactive gases reach the surface of the substrate in atomic form, after dissociation of the gas molecules in the plasma, in front of the substrate. These atoms were regarded as ,active" and ready available to contribute to the crystal growth. We considered that the $M G_{1}$ and $M G_{2}$ molecules sputtered from the target, reach the substrate in the same form, without dissociation.

We assumed that the condensation surface is composed of the surface of the substrate and the surface fraction of the vacuum chamber situated in the range of the sputtered particles.

The surface of the chamber is present in the model as a getter pump with adsorption efficiencies $\alpha_{g_{1}}$ and $\alpha_{g_{2}}$ (1), corresponding to $G_{1}$ and $G_{2}$.

$$
\alpha_{g 1,2}=\frac{N r \text { of adsorbed_gas_molecules }}{\text { Nr incident gas molecules }}
$$

The following notation has been used in the article:

$n_{M G i}$ - number of metal atoms in the molecule formed with the gas $G_{i}$ (ex. $n_{M G 1}=1$ in $\mathrm{TiO}_{2}, n_{M G 2}=1$ in $\left.T i N\right)$;

$n_{\text {GiM }}$ - number of gas atoms in a binary molecule (ex. $n_{G 1 M}=2$ in $\mathrm{TiO}_{2}$, $n_{G 2 M}=1$ in $\left.T i N\right)$;

$n_{G i G i}$ - number of gas atoms in a gas molecule;

$n_{M G 1 G 2}$ - number of metal atoms in the $M G_{1} G_{2}$ compound (ex. $n_{M G 1 G 2}=1$ in the $\mathrm{TiO}_{2} N$ molecule); in this model, it has been assumed that $n_{M G 1 G 2}=n_{M G 1}=n_{M G 2}$;

$x, y$-number of $G_{1}$ and $G_{2}$ atoms in the $M G_{1} G_{2}$ compound;

$k_{B}=1.38 e-23\left[\frac{J}{K}\right]$ - the Boltzmann constant;

$N_{A}=6.023 e 26\left[\frac{1}{\mathrm{kmol}}\right]$ - the Avogadro constant;

$e=1.6 e-19[C]-$ the electron charge;

$R=8310\left[\frac{J}{k m o l K}\right]$ - the universal gas constant; 
$M_{G 1}$ - mass of the $G_{1}$ gas molecule $\left(O_{2}\right)$;

$M_{G 2}$ - mass of the $G_{2}$ gas molecule $\left(N_{2}\right)$;

$N_{M}$ - the surface density of the metal atoms ( $T i$ );

$T$ - the absolute temperature;

$V$ - the volume of the vacuum chamber;

$A_{t}$ - the target area;

$A_{c}$ - the condensation area;

$A_{s}$ - the substrate area;

$A_{g}$ - the getter area;

$S_{G 1}$ - the pumping speed of the reactive gas $G_{1}\left(O_{2}\right)$;

$S_{G 2}$ - the pumping speed of the reactive gas $G_{2}\left(N_{2}\right)$;

$\eta_{M}$ - sputtering efficiency of the metal (number of $T i$ atoms sputtered by an incident $\mathrm{Ar}^{+}$ion);

$\eta_{M G i}$ - sputtering efficiency of the $M G_{i}$ compound;

$\alpha_{t G i}$ - sticking coefficient of the $G_{i}$ reactive gas atom to the surface of the target;

$\alpha_{g G i}$ - the gettering efficiency of the $G_{i}$ reactive gas atom;

$\boldsymbol{\alpha}_{\mathrm{s}}$ - the matrix of sticking coefficients on the surface of the substrate (the $\alpha_{s i j}$ element of this matrix is the sticking coefficient of a particle type $i$ to the area type $j$ of the substrate- see Table 1);

$\mathbf{c}_{\mathbf{s}}=\left[\begin{array}{llllllll}c_{M G 1 G 2} & c_{M G 1 a} & c_{M G 2 a} & c_{G 1 a} & c_{G 2 a} & c_{M G 1 p} & c_{M G 2 p} & c_{M}\end{array}\right]$ - the elements of the $\mathbf{c}_{\mathrm{s}}$ matrix represent the per unit area (the number of covered metal atoms) of the compounds corresponding to the subscripts.

Table 1: Interpretation of subscript i of the $\alpha_{s i j}$ sticking coefficient

\begin{tabular}{|c|c|c|c|c|c|}
\hline Row number $(i)$ & 1 & 2 & 3 & 4 & 5 \\
\hline Particle type & $M G_{1}$ & $M G_{2}$ & $G_{1}$ & $G_{2}$ & $M$ \\
\hline
\end{tabular}


Table 2: Interpretation of subscript $\mathrm{j}$ of the $\alpha_{s i j}$ sticking coefficient

\begin{tabular}{|c|c|c|c|c|c|c|c|c|}
\hline $\begin{array}{c}\text { Column } \\
\text { number }(j)\end{array}$ & 1 & 2 & 3 & 4 & 5 & 6 & 7 & 8 \\
\hline $\begin{array}{c}\text { Substrate } \\
\text { fraction }\end{array}$ & $\theta_{S M G 1 G 2}$ & $\theta_{S M G 1 a}$ & $\theta_{S M G 2 a}$ & $\theta_{s G 1 a}$ & $\theta_{S G 2 a}$ & $\theta_{S M G 1 p}$ & $\theta_{S M G 2 p}$ & $\theta_{S M}$ \\
\hline
\end{tabular}

The block diagram shown in Fig. 2 presents the main tasks performed by the model introduced in this paper.

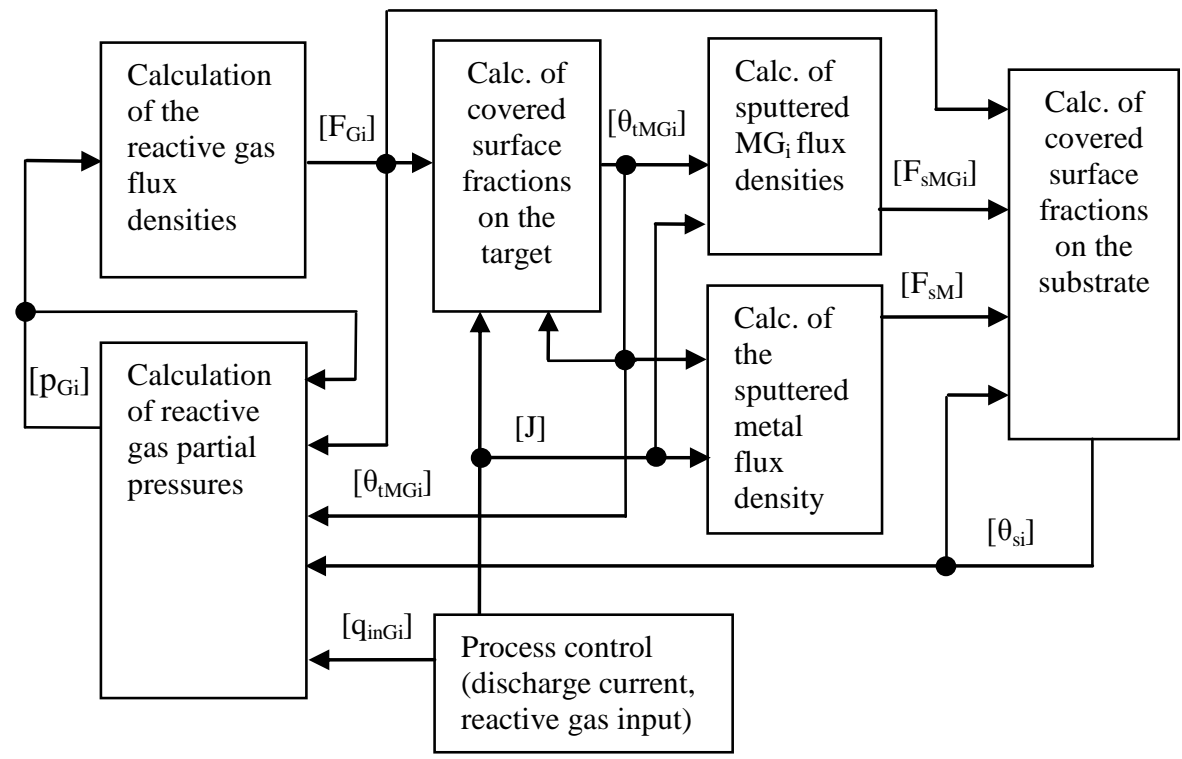

Figure 2: The main components of the two-reactive-gas sputtering model

\section{Particle flux densities and the dynamics of the target coverage}

The dynamics of the areas covered by different compounds on both the surface of the target and of the substrate is determined by the incident fluxes of particles, by the fluxes of particles removed via sputtering, and by the complex phenomena on these surfaces. These include the formation of chemical compounds, the crystal growth, and the segregation of some compounds on the grain boundary and the migration of particles.

On the surface of the target, the sputtering effect is produced by the incident flux of $\mathrm{Ar}^{+}$ions, while the fluxes of reactive gases result in chemical reactions, 
i.e. target poisoning. It was assumed that metal atoms are sputtered from the surface of the target, and metal-gas compounds can be sputtered as well.

On the surface of the substrate, the sputtered particles produce incident fluxes, which participate in the process of thin film formation, along with the reactive gas fluxes resulting from the atmosphere created in the chamber.

The flux density $J$ of the sputtering $\mathrm{Ar}^{+}$ions is calculated from the discharge current $I$, neglecting the secondary electron emission and the ion current of the reactive gases, according to (2).

$$
J=\frac{I}{e A_{t}}
$$

The atomic flux densities $F_{\text {Gia }}$ of the reactive gases are calculated using results of the kinetic gas theory:

$$
F_{G i a}=\frac{n_{G i G i} p_{G i}}{\sqrt{2 \pi k_{B} T M_{G i}}},
$$

where $i=1$ for oxygen and $i=2$ for nitrogen.

On the substrate, the $F_{S M G i}$ flux densities of the $M G_{i}$ particles sputtered from the target are calculated using equation (4), assuming that these are uniformly distributed on the condensation area $A_{c}$.

$$
F_{s M G i}=J * \eta_{M G i} * \theta_{t M G i} * \frac{A_{t}}{A_{c}}
$$

The same approach stands for the $F_{s M}$ flux density of the metal atoms sputtered from the target

$$
F_{s M}=J * \eta_{M} * \theta_{t M} * \frac{A_{t}}{A_{c}}=J * \eta_{M} *\left(1-\theta_{t M G 1}-\theta_{t M G 2}\right) * \frac{A_{t}}{A_{c}}
$$

In the case of the target, it is assumed that the $G_{i}$ gas atoms adhere only to the metallic fraction $\theta_{t M}$ of the surface, forming $M G i$ compounds (poisoning the target).

The number of $M G i$ molecules formed on the unit area of the target in a second (the rate of increment of the $N_{t M G i}$ surface density of $M G i$ molecules) is

$$
\dot{N}_{t M G i}^{+}=\frac{n_{G i G i}}{n_{G i M}} F_{G i a} \alpha_{t G i}\left(1-\sum_{i} \theta_{t M G i}\right) .
$$

The number of $M G i$ molecules removed by sputtering from the unit area of the target (the rate of decrement of $N_{t M G i}$ ) is 


$$
\dot{N}_{t M G i}^{-}=A_{s} J \eta_{G i} \theta_{t M G i} .
$$

From the equilibrium of these processes, it results $\dot{N}_{t M G i}^{+}=\dot{N}_{t M G i}^{-}$, i.e.

$$
\frac{n_{G i G i}}{n_{G i M}} F_{G i a} \alpha_{t G i}\left(1-\sum_{i} \theta_{t M G i}\right)=J \eta_{M G i} \theta_{t M G i}
$$

Usually, $\eta_{M G i}<<\eta_{M}$, i.e. the sputtering yield of the metal atoms is much bigger than that of the $M G_{i}$ compounds.

The dynamics of the covered fraction $\theta_{t M G i}$ is described by the equations

$$
\left\{\begin{array}{l}
\dot{\theta}_{t M G i}=\frac{c_{M G i p}}{N_{M}}\left[\frac{\alpha_{t G i} F_{G i a}}{n_{G i M}}\left(1-\sum_{i} \theta_{t M G i}\right)-J \eta_{M G i} \theta_{t M G i}\right] . \\
\theta_{t M G i}=\theta_{t M G i}\left(t_{0}\right)+\int_{t 0}^{t} \dot{\theta}_{t M G i} d t
\end{array}\right.
$$

\section{Macroscopic modelling of the thin film deposition process}

During the thin film deposition process, different fractions of the substrate surface are covered by atoms provided by the incident gas fluxes, by particles sputtered from the surface of the target and by different compounds formed during the crystal growth.

The aim is the deposition of $M G_{1} G_{2}$, and an optimal process control has to enassure atomic fluxes corresponding to the stoichiometry of this compound.

The model introduced in this paper considers the continuous emergence and disappearance of areas covered by $M, G_{1 a}, G_{2 a}, M G_{1 a}, M G_{2 a}, M G_{1 p}, M G_{2 p}$ and $M G_{1} G_{2}$, on each of the $\theta_{s M}, \theta_{s M G 1 G 2}, \theta_{s M G 1 a}, \theta_{s M G 2 a}, \theta_{s M G 1 p}, \theta_{s M G 2 p}, \theta_{s G 1 a}$ and $\theta_{s G 2 a}$ fractions. Thus, the „local” processes taking place on each of the fractions contribute to the global evolution of the different fractions.

Let us use the following notation:

$N_{s i}^{j}$ - the surface density of the molecules of type $i$, emerging on the fraction $\theta_{s j}$ of the substrate, and thus contributing to the decrease of the fraction $\theta_{s j}$ and to the increase of the fraction $\theta_{s i}$;

$F_{s i}$ - the flux density of the particles type $i$ on the substrate (the same for every fraction); 
$R_{s i}^{j}$ - the surface density of the molecules type $i$, emerging on the fraction $\theta_{s j}$ of the substrate by reaction between different incident particles or by reaction between incident particles and the molecules type $j$.

Thus, the time derivative of $N_{s i}^{j}$ results:

$$
\dot{N}_{s i}^{j}=F_{s i}+\dot{R}_{s i}^{j}
$$

Due to these emerging molecules, the corresponding surface fraction is subject to transition to other types of fractions, characteriszed by the rate of growth $\dot{\theta}_{s i}^{j}$ (i.e. time derivative of the surface fraction type $i$ emerging on the surface type $j$ ):

$$
\dot{\theta}_{s i}^{j}=\left\{\begin{array}{lll}
\frac{c_{i}}{N_{M}} \dot{N}_{s i}^{j} & \text { if } & i \neq j \\
0 & \text { if } & i=j
\end{array}\right.
$$

The weighted sum (12) of the rates of growth of fractions type $i$ yields the time derivative of $\theta_{s i}$.

$$
\dot{\theta}_{s i}=\sum_{j} \theta_{s j} \dot{\theta}_{s i}^{j}
$$

The evolution of the substrate surface fractions is found by integration (13), subject to the condition $\sum_{i} \theta_{s i}=1$.

$$
\theta_{s i}=\left\{\theta_{s i}\left(t_{0}\right)+\int_{t_{0}}^{t} \dot{\theta}_{s i} d t \mid \sum_{i} \theta_{s i}=1\right\}
$$

The number of particles available for the formation of different compounds depends on the incident fluxes and on the sticking coefficients.

It is assumed that the ternary compound $M G_{1} G_{2}$ is formed from incident atoms and "active" binary molecules present on the surface, or directly from incident atoms to the extent of their availability in quantities corresponding to the required stoichiometry $M G_{1 x} G_{2 y}$.

The particles in excess of this ternary stoichiometry form binary compounds, and the atoms still in excess of the possible binary stoichiometries are deposited as atomic layers.

In order to illustrate this reasoning, the relation (10) is detailed below (Eqs. (14), (15), (16), (17) and (18)) for the case of the fraction $\theta_{s M G 1 G 2}$. In the general notation $N_{s i}^{j}$, we use the particular $j=M G_{1} G_{2}$ upper index. Thus, for 
example, in the case of the molecules type $M G_{1 a}$ emerging on $\theta_{S M G 1 G 2}$, the lower index becomes $i=M G_{1 a}$, and the surface density is denoted by $N_{s M G 1 a}^{M G 1 G 2}$.

If $\alpha_{51} F_{s M}<\alpha_{31} \frac{F_{s G 1 a}}{x}$ and $\alpha_{51} F_{s M}<\alpha_{41} \frac{F_{s G 2 a}}{y}$

$$
\left\{\begin{array}{l}
\dot{N}_{s M G 1 G 2}^{M G 1 G 2}=\alpha_{51} F_{s M} \\
\dot{N}_{s G 1 a}^{M G 1 G 2}=\alpha_{31} F_{s G 1 a}-x \alpha_{51} F_{s M} \\
\dot{N}_{s G 2 a}^{M G 1 G 2}=\alpha_{41} F_{s G 2 a}-y \alpha_{51} F_{s M}
\end{array}\right.
$$

Else if $\alpha_{31} \frac{F_{s G 1 a}}{x}<\alpha_{51} F_{s M}<\alpha_{41} \frac{F_{s G 2 a}}{y}$

$$
\left\{\begin{array}{l}
\dot{N}_{s M G 1 G 2}^{M G 1 G 2}=\alpha_{31} \frac{F_{s G 1 a}}{x} \\
\dot{N}_{s M G 2 a}^{M G 1 G 2}=\alpha_{51} F_{s M}-\alpha_{31} \frac{F_{s G 1 a}}{x} \\
\dot{N}_{s G 2 a}^{M G 1 G 2}=\alpha_{41} F_{s G 2 a}-y \alpha_{51} F_{s M}
\end{array}\right.
$$

Else if $\alpha_{31} \frac{F_{s G 1 a}}{x}<\alpha_{41} \frac{F_{s G 2 a}}{y}<\alpha_{51} F_{s M}$

$$
\left\{\begin{array}{l}
\dot{N}_{s M G 1 G 2}^{M G 1 G 2}=\alpha_{31} \frac{F_{s G 1 a}}{x} \\
\dot{N}_{s M G 2 a}^{M G 1 G 2}=\frac{1}{y} \alpha_{41} F_{s G 2 a}-\frac{1}{x} \alpha_{31} F_{s G 1 a} \\
\dot{N}_{s M}^{M G 1 G 2}=\alpha_{51} F_{s M}-\alpha_{41} \frac{F_{s G 2 a}}{y}
\end{array}\right.
$$

Else if $\alpha_{41} \frac{F_{s G 2 a}}{y}<\alpha_{51} F_{s M}<\alpha_{31} \frac{F_{s G 1 a}}{x}$ 


$$
\left\{\begin{array}{l}
\dot{N}_{s M G 1 G 2}^{M G 1 G 2}=\alpha_{41} \frac{F_{s G 2 a}}{y} \\
\dot{N}_{s M G 1 a}^{M G 1 G 2}=\alpha_{51} F_{s M}-\alpha_{41} \frac{F_{s G 2 a}}{y} \\
\dot{N}_{s G 1 a}^{M G 1 G 2}=\alpha_{31} F_{s G 1 a}-x \alpha_{51} F_{s M}
\end{array}\right.
$$

Else if $\alpha_{41} \frac{F_{s G 2 a}}{y}<\alpha_{31} \frac{F_{s G 1 a}}{x}<\alpha_{51} F_{s M}$

$$
\left\{\begin{array}{l}
\dot{N}_{s M G 1 G 2}^{M G 1 G 2}=\alpha_{41} \frac{F_{s G 2 a}}{y} \\
\dot{N}_{s M G 1 a}^{M G 1 G 2}=\frac{1}{x} \alpha_{31} F_{s G 1 a}-\frac{1}{y} \alpha_{41} F_{s G 2 a} \\
\dot{N}_{s M}^{M G 1 G 2}=\alpha_{51} F_{s M}-\alpha_{31} \frac{F_{s G 1 a}}{x}
\end{array}\right.
$$

The steady state of the reactive gas quantities inside the sputtering chamber means relates to the equilibrium between the reactive gas admission, evacuation by pumping, release from and adsorption to different surfaces.

The dynamics of the partial pressures is related to the dynamics of the masses by means of the ideal gas law (19).

$$
\left\{\begin{array}{l}
\dot{p}_{G i}=\frac{R T}{V N_{A} M_{G i}} \dot{m}_{G i} \\
p_{G i}=p_{G i}\left(t_{0}\right)+\int_{t 0}^{t} \dot{p}_{G i} d t
\end{array},\right.
$$

where $\dot{m}_{G i}$ is the time derivative of the mass of $G_{i}$ (Eq. (24)).

The inlet mass flow of each gas is controlled and is denoted by $q_{\text {in } i i}$.

The pumping mass flow is proportional to the partial pressure of the gas

$$
q_{p G i}=S_{G i} p_{G i}
$$

The gases are adsorbed to the walls of the chamber (getter effect), deposited on the target (poisoning effect) and on the substrate (thin film growth).

The mass flow of the gettered gas is given by the equation

$$
q_{g G i}=M_{G i} \frac{F_{G i a}}{n_{G i G i}} \alpha_{g G i} A_{g} .
$$


The mass flow of the gas adsorbed to the metallic fraction of the target surface is

$$
q_{t G i}=M_{G i} \frac{F_{G i a}}{n_{G i G i}} \alpha_{t G i} A_{t}\left(1-\sum_{j} \theta_{t j}\right) .
$$

The mass flow of the gas adsorbed to different fractions of the substrate is

$$
q_{s G i}=M_{G i} \frac{F_{G i a}}{n_{G i G i}} A_{s} \sum_{j} \alpha_{s i j} \theta_{s j} .
$$

Thus,

$$
\dot{m}_{G i}=q_{i n G i}-q_{p G i}-q_{g G i}-q_{t G i}-q_{s G i} .
$$

Given the input quantities provided by the controller (i.e. the discharge current and the inlet gas flows), and the dynamic model of the sputtering process (Eqs. (9) to (13) and Eqs. (19) to (24) corresponding to the relationships shown in Fig. 2), it becomes possible to determine the evolution in time of the areas covered by different compounds both on the target and on the substrate. It is also possible to determine the evolution of the partial pressures.

\section{Simulation results}

In order to demonstrate that the model is able to describe the nonlinear behaviour of the sputtering system, we present simulation results based on hypothetical model parameters. Figures 3 to 7 show the Matlab Simulink simulation results for the following parameters:

-The sputtered metal is $T i\left(N_{M}=1.62 \times 10^{19} 1 / \mathrm{m}^{2}\right)$;

- $G_{1}$ is oxygen $\left(O_{2}, n_{G 1 G 1}=2, M_{G 1}=53.12 \times 10^{-27} \mathrm{~kg}\right)$;

- $G_{2}$ is nitrogen $\left(N_{2}, n_{G 2 G 2}=2, M_{G 2}=46.48 \times 10^{-27} \mathrm{~kg}\right)$;

-Binary compounds: $\mathrm{TiO}_{2}, \mathrm{TiN}\left(n_{M G 1}=1, n_{M G 2}=1, n_{G 1 M}=2, n_{G 2 M}=1\right)$;

-Ternary compound: $\mathrm{TiO}_{2} N\left(n_{M G 1 G 2}=1, x=2, y=1\right)$;

$-T=300 \mathrm{~K}$;

$-V=80 \times 10^{-3} \mathrm{~m}^{3}$;

- $A_{t}=0.84 \times 10^{-2} \mathrm{~m}^{2}, A_{c}=0.22 \mathrm{~m}^{2}, A_{s}=0.22 \mathrm{~m}^{2}, A_{g}=0.3 \mathrm{~m}^{2}$;

$-S_{G 1}=92 \times 10^{-7} m \bullet s, S_{G 2}=72.9 \times 10^{-7} m \bullet s$;

$-\eta_{M}=5, \eta_{M G 1}=0.2, \eta_{M G 2}=0.1$;

$-\alpha_{t G 1}=1, \alpha_{t G 2}=1, \alpha_{g G 1}=0, \alpha_{g G 2}=0$; 


$$
\begin{aligned}
& \theta_{s M G_{1} G_{2}}, \theta_{s M G_{1 a}}, \theta_{s M G_{2 a}}, \theta_{s G_{1 a}}, \theta_{s G_{2 a}}, \theta_{s M G_{1 D}}, \theta_{s M G_{2 D}}, \theta_{s M} \\
& \boldsymbol{\alpha}_{s}=\left[\begin{array}{cccccccc}
0 & 0 & 0 & 0 & 0 & 0 & 0 & 0 \\
0 & 0.2 & 0.2 & 0.3 & 0.2 & 0 & 0 & 0.4 \\
0 & 1 & 0 & 0 & 0 & 0 & 0 & 1 \\
0 & 0 & 1 & 0 & 0 & 0 & 0 & 1 \\
1 & 1 & 1 & 1 & 1 & 1 & 1 & 1
\end{array}\right] \begin{array}{c}
M G_{1} \\
M G_{2} \\
G_{1} \\
G_{2} \\
M
\end{array} \\
& -\mathbf{c}_{\mathbf{s}}=\left[\begin{array}{llllllll}
4 & 3 & 2 & 1 & 1 & 3 & 2 & 1
\end{array}\right] \text {. }
\end{aligned}
$$

The elements of the $\mathbf{c}_{\mathbf{s}}$ matrix represent the per unit area (the number of covered metal atoms) of the compounds corresponding to the subscripts.
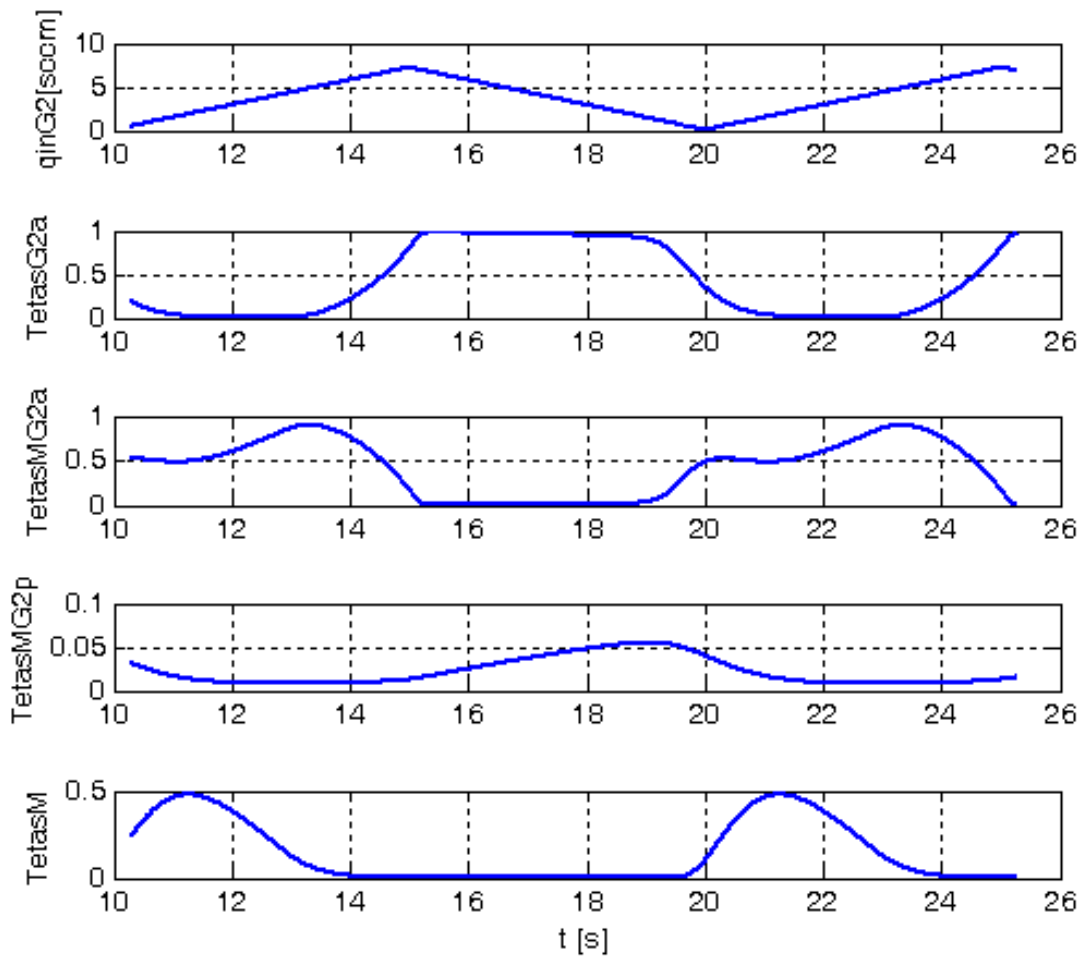

Figure 3: Evolution of the substrate fractions in the case of the admission of a single gas $\left(G_{2}\right)$, with a periodic variation of its flow rate (uppermost diagram) 


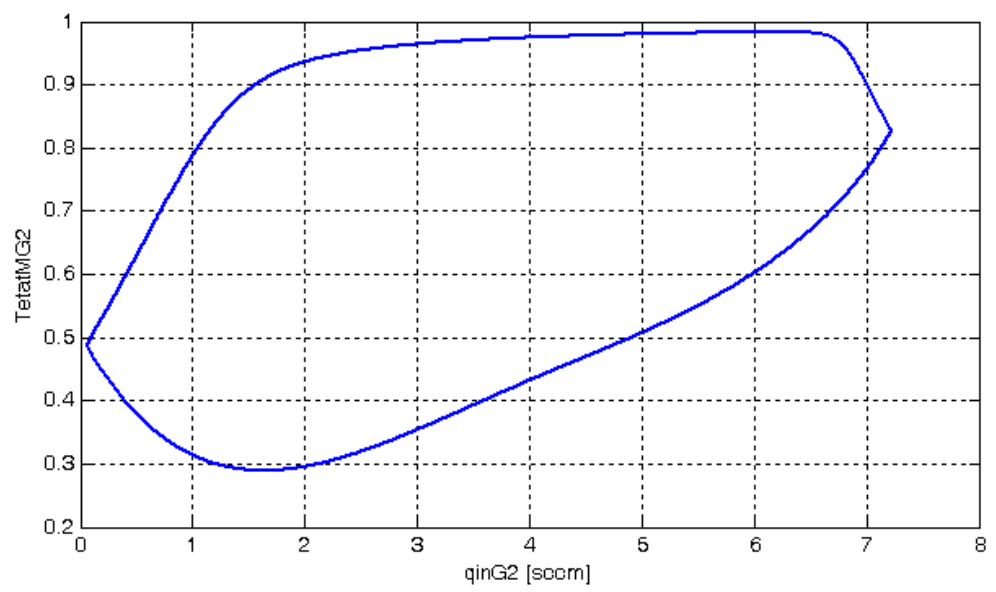

Figure 4: The dynamic hysteresis cycle of the target fraction covered by the $M G_{2}$ ( TiN ) compound, in the case of the admission of a single gas $\left(G_{2}\right)$, with a flow rate variation period of $10 \mathrm{~s}$

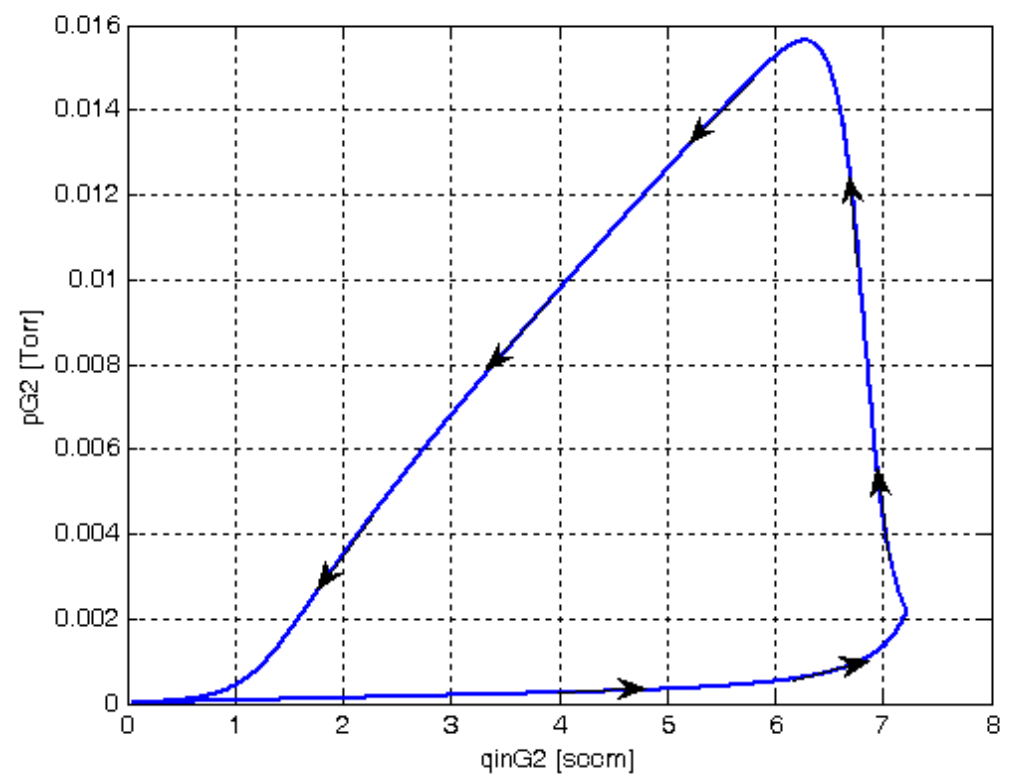

Figure 5: The dynamic hysteresis cycle of the $G_{2}$ gas pressure, in the case of the admission of a single gas $\left(N_{2}\right)$, with a flow rate variation period of $10 \mathrm{~s}$ 

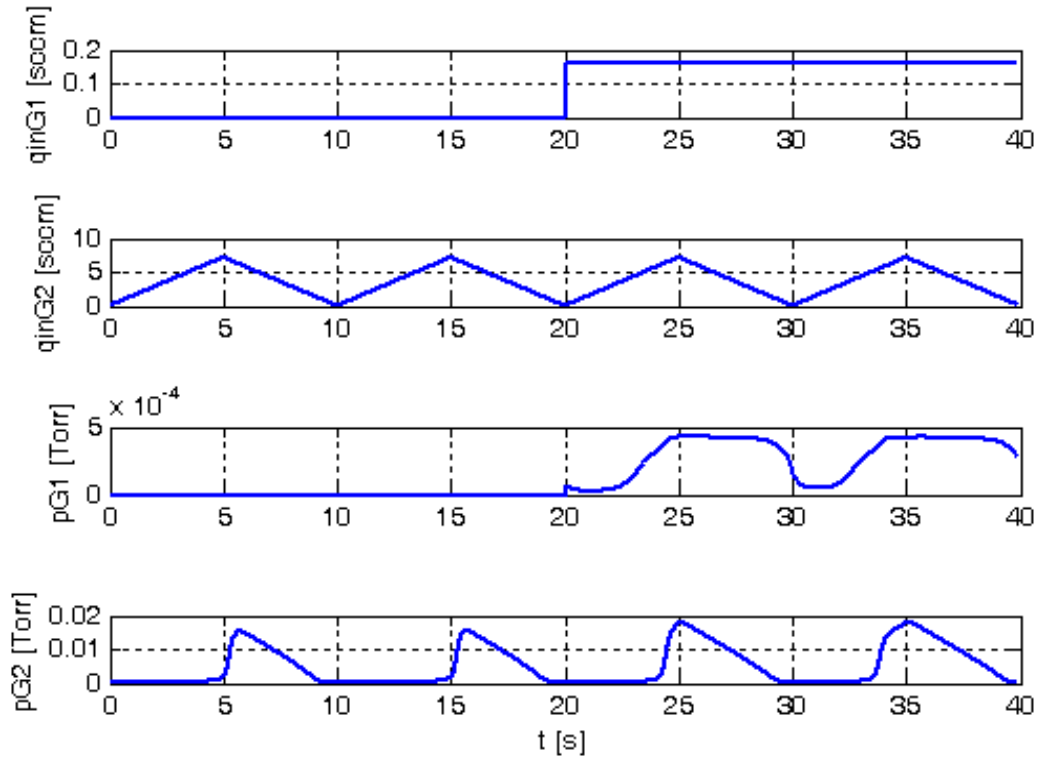

Figure 6: The admission of $G_{1}\left(O_{2}\right)$ has a strong influence on the partial pressure of $G_{2}\left(N_{2}\right)$

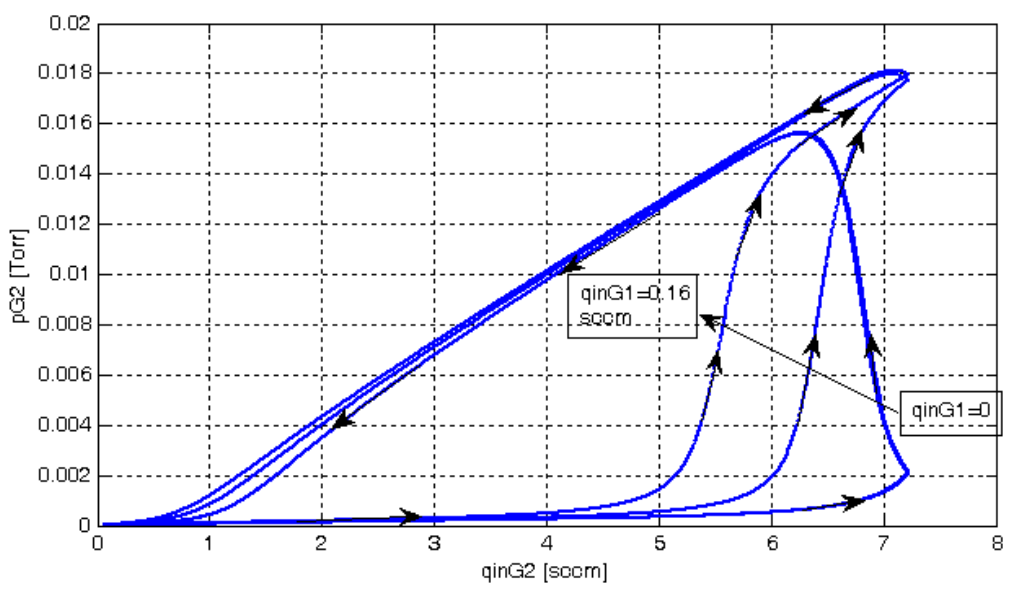

Figure 7: The influence of a small ( $0.16[\mathrm{sccm}]) G_{1}\left(\mathrm{O}_{2}\right)$ gas flow step on the trajectory described in the $q_{i n G 2}-p_{G 2}$ space ( $N_{2}$ flow rate and partial pressure) 


\section{Conclusions}

The dynamic model introduced in this paper has been developed to describe the essential macroscopic phenomena that accompany the DC magnetron sputtering and thin film deposition process in the presence of two or more reactive gases.

We defined state variables of the model, which are directly related to the stoichiometry of the compounds formed on the surface of the substrate. These state variables offer an insight into the satisfaction of the conditions required for the growth of crystals with the required stoichiometry.

In order to prepare the stoichiometry control of the deposition process, our next target is the identification of the model parameters and the practical validation of the model.

\section{References}

[1] Berg, S., Nyberg, T., Blom, H.-O., and Nender, C., "Computer modeling as a tool to predict deposition rate and film composition in the reactive sputtering process", J. Vac. Sci. Technol. A 16(3)., May/Jun 1998, pp. 1277-1285.

[2] Berg, S., Nender, C., „Modeling of Mass Transport and Gas Kinetics of the Reactive Sputtering Process”, Journal de Physique IV, Colloque C5, supplément au Journal de Physique II, Volume 5, juin 1995, pp. 45-54.

[3] Berg, S., Katardijev, I. V., „Preferential sputtering effects in thin film processing”, J. Vac. Sci. Technol. A 17(4)., Jul/Aug 1999, pp. 1916-1925.

[4] Jonsson, L. B., Nyberg, T., and Berg, S., "Target compound layer formation during reactive sputtering”, J. Vac. Sci. Technol. A 17(4), Jul/Aug 1999, pp. 1827-1831.

[5] Güttler, D., Abendroth, B., Grötzschel, R., Möller, W., and Depla, D., „Mechanisms of target poisoning during magnetron sputtering as investigated by real-time in situ analysis and collisional computer simulation”, Dept. Solid State Sciences, Univ. of Ghent, Annual Report IIM 2004, FZR-427, pp. 34-37.

[6] Kubart, T., Polcar, T., Kappertz, O., Parreira, N., Nyberg, T., Berg, S., and Cavaleiro, A., „Modelling of magnetron sputtering of Tungsten Oxide with reactive gas pulsing”, Plasma Processes and Polymers, 2007, 4, WILEY-VCH Verlag GmbH \& Co. KGaA, Weinheim, pp. S522-S526, DOI: 10.1002/ppap.200731301.

[7] Görgy, K., „Cercetări privind dezvoltarea unor electrotehnologii pentru depunerea straturilor metalice subţiri”, Teză de doctorat, Universitatea Tehnică din Cluj-Napoca, 2010.

[8] Christie D., J., „Making magnetron sputtering work: Modelling reactive sputtering dynamics, Part 1", SVC Bulletin, Fall 2014, pp. 24-27.

[9] Christie D., J., „Making magnetron sputtering work: Modelling reactive sputtering dynamics, Part 2", SVC Bulletin, Spring 2015, pp. 30-33.

[10] Christie D., J., „Making magnetron sputtering work: Modelling reactive sputtering dynamics, Part 3", SVC Bulletin, Summer 2015, pp. 38-41.

[11] Carlsson, P., Nender, C., Barankova, H., and Berg, S., „Reactive sputtering using two reactive gases, experiments and computer modeling", J. Vac. Sci. Technol. A 11(4), Jul/Aug 1993, pp. 1534-1539. 
[12] Sproul, W. D., Christie, D. J., Carter, D. C., Berg, S., and Nyberg, T., „Control of the reactive sputtering process using two reactive gases", 46'th Annual Technical Conference Proceedings, Society of Vacuum Coaters, 2003, pp. 98-103.

[13] Carter, D. C., Sproul, W. D., and Christie, D. J., „Effective closed-loop control for reactive sputtering using two reactive gases", 47'th Annual Technical Conference Proceedings, Society of Vacuum Coaters, 2004, pp. 37-43.

[14] Kubart, T., Trinh, D. H., Liljeholm, L., Hultman, L., Högberg, H., Nyberg, T., and Berg, S., „Experiments and modeling of dual reactive magnetron sputtering using two reactive gases”, J. Vac. Sci. Technol. A 26(4), Jul/Aug 2008, pp. 565-570.

[15] Papp, S., "Optimizarea controlului automat al pulverizării catodice în procesul de obţinere a straturilor subţiri”, Teză de doctorat, Universitatea Transilvania din Braşov, 2012. 